\title{
Nonspecific enhancement of gene expression by compounds identified in high-throughput cell-based screening
}

\author{
Steven C. Cunningham, Byungwoo Ryu, Taylor A. Sohn, and Scott E. Kern \\ The Johns Hopkins University School of Medicine, Baltimore, MD, USA
}

BioTechniques 37:120-122 (July 2004)

Cell-based reporter assays are increasingly used for high-throughput screening of chemical libraries. However, reporter plasmids are subject to artifacts arising from any source of variance in promoter activity. For example, some common vectors have been found to contain occult steroid- or transcription-factor-responsive elements (1-4). Because reporter plasmids are frequently used in molecular and cellular biology to report pathway activity after treatment with experimental agents, it would be helpful for investigators to be aware of such effects, how often they occur, in which classes of structures they are often observed, in which direction the activity is likely to shift, and how subtle or robust is the artifact. In addition, nontranscriptional artifacts of compound screening include contaminants that reflect or absorb light in colorimetric assays, autofluorescence of cells, plastics, and media that decreases the signal-to-noise ratio in fluorometric assays and decreases the aggregates of small molecules that serve as inhibitors of cell-surface receptors (5).

We recently reported the novel histone deacetylase inhibitor (HDACI) Scriptaid, which was identified through screening of a compound library on a Smad4-reporter (Smad4-R) cell line (6). Scriptaid, like trichostatin-A (TSA) (7), is a hydroxymate that nonspecifically up-regulates transcription of multiple genes by inhibiting the deacetylation of lysine residues on histones. Here, we extend our screening experiments to explore additional agents, found among diverse compounds and among drugs currently in clinical use, that share the characteristic of indiscriminately augmenting gene expression.

We screened a 16,320-compound library (DIVERSet E; ChemBridge, San Diego, CA, USA) and an 880-drug library (i.e., agents previously tested for acceptable bioavailability and toxicity profiles: Monotarget Set; Prestwick
Chemical, Washington, DC, USA). Each compound in the screen was diluted in dimethylsulfoxide (DMSO) at $0.5 \mathrm{mg} / \mathrm{mL}$ (compounds) or $1 \mathrm{mg} / \mathrm{mL}$ (drugs). Cells containing transcriptional reporter systems (Table 1) were plated, incubated with each compound at $2 \mu \mathrm{g} /$ $\mathrm{mL}$, and tested as described (6).

The 17,220 agents $(16,320$ compounds and 880 drugs) were screened initially against Smad4-R cells and p53reporter (p53-R) RKO cells (8). There were 14 agents (structures shown in supplementary Figure S1, found on the BioTechniques web site at http://www. BioTechniques.com/July2004/CunninghamSupplementary.html that activated both Smad4-R and p53-R cell lines more than 2-fold and were therefore chosen as a panel to test on six other available reporter plasmid systems over a concentration range of 1 to $10 \mu \mathrm{g} / \mathrm{mL}$. Seven compounds $(0.04 \%)$ and five drugs $(0.57 \%)$ indiscriminately elevated the activity of multiple reporters; one of the compounds was Scriptaid. All reporter systems were up-regulated upon treatment except MDA-kb2, containing the mouse mammary tumor virus (MMTV) promoter, which is known to be repressed by HDACIs (9). Eleven agents activated two or more reporter systems; eight activated three or more (Table 2) (activation was defined as a greater-than two-fold induction). This effect was independent of type of reporter [e.g., luciferase-based versus fluorescence-based (Table 1)] and the type of transfection [transient versus stable (data from Ref-

Table 1. Plasmid Reporter Systems

\begin{tabular}{|c|c|c|c|c|c|c|}
\hline Cell Type & References & Reporter Name & Reported & Emission & Promoter & Enhancer \\
\hline HPC (PANC-1) & 6 & Smad4-R & Smad4 function & FF-luc & $\min$ SV40 & $6 \mathrm{SBE}$ \\
\hline HCC (RKO) & 8 & p53-R & p53 binding & FF-luc & $\min$ SV41 & \\
\hline HCC (HCT116) & 11 & p53-R & p53 binding & FF-luc & & \\
\hline HCC (DLD-1) & 12 & DLD/BFP & constitutive & Fluor & CMV & \\
\hline $\mathrm{CHO}$ & 13 & CHOAA8-luc-tet-off & constitutive & FF-luc & CMV & \\
\hline MEF (NIH/3T3) & 14 & Shh Light 2 & Hedgehog protein & FF-luc & & \\
\hline MEF (NIH/3T3) & 14 & Shh Light 2 & constitutive & Ren-luc & HSV-TK & \\
\hline HBC (MDA-kb-2) & 15 & MDA-kb2 & AR, GR stimulation & FF-luc & MMTV & \\
\hline \multicolumn{7}{|c|}{$\begin{array}{l}\text { Eight different reporter plasmids stably transfected into seven cell lines were used to test a panel of agents that activated the } \\
\text { Smad4-reporter system. CHO AA8 Tet-Off cell line was obtained from BD Biosciences Clontech, San Jose, CA, USA. MDA- } \\
\text { kb2 cell line was obtained from ATCC, Manassas, VA, USA. Other cell lines were gifts as described in the Acknowledgments } \\
\text { section or engineered in our laboratory as referenced. } \\
\text { Abbreviations: HPC, human pancreas carcinoma; HCC, human colon carcinoma; MEF, mouse embryonal fibroblasts; HBC, } \\
\text { human breast carcinoma; CHO, Chinese hamster ovary; AR: androgen receptor; GR: glucocorticoid receptor; SV40, simian } \\
\text { virus 40; CMV, cytomegalovirus; HSV-TK, herpes simplex virus thymidine kinase; MMTV, mouse mammary tumor virus. }\end{array}$} \\
\hline
\end{tabular}


Table 2: Fold Activation Over DMSO Control

\begin{tabular}{|c|c|c|c|c|c|c|c|c|}
\hline \multirow[b]{2}{*}{ Agent Tested } & \multirow[b]{2}{*}{$\begin{array}{c}\text { Reporters } \\
\text { Activated } \\
\text { (No.) }\end{array}$} & \multicolumn{7}{|c|}{ Reporter Systems } \\
\hline & & $\begin{array}{c}1 \\
\text { Smad4R }\end{array}$ & $\begin{array}{c}2 \\
\text { RKO p53R }\end{array}$ & $\begin{array}{c}3 \\
\text { HCT116 p53R }\end{array}$ & $\begin{array}{c}4 \\
\text { DLD/BFP }\end{array}$ & $\begin{array}{c}5 \\
\text { CHO-AA8 }\end{array}$ & $\begin{array}{c}6 \\
\text { Shh FF }\end{array}$ & $\begin{array}{c}7 \\
\text { Shh REN }\end{array}$ \\
\hline Quinacrine & 6 & $4.1 @ 2$ & $18.9 @ 2$ & $16.7 @ 5$ & $1.3 @ 5$ & $23.1 @ 10$ & $14.5 @ 5$ & $2.4 @ 1$ \\
\hline PTT & 3 & $1.8 @ 5$ & $2.2 @ 2$ & $1.9 @ 5$ & $1.5 @ 5$ & $10.8 @ 10$ & $4.2 @ 5$ & $1.4 @ 1$ \\
\hline Piperine & 2 & $1.7 @ 5$ & $1.8 @ 1$ & $1.9 @ 10$ & $1.4 @ 5$ & $4.2 @ 10$ & $1.8 @ 1$ & $3.1 @ 1$ \\
\hline Apigenin & 4 & $1.6 @ 2$ & $2.6 @ 5$ & $2.4 @ 10$ & $1.2 @ 1$ & $4.6 @ 10$ & $1.6 @ 1$ & $2.5 @ 1$ \\
\hline Hydralazine & 1 & $1.7 @ 5$ & $1.6 @ 2$ & $1.6 @ 10$ & $1.4 @ 5$ & $1.4 @ 10$ & $1.6 @ 1$ & $2.3 @ 1$ \\
\hline 5110235 & 1 & $1.7 @ 5$ & $1.8 @ 2$ & $1.8 @ 10$ & $1.4 @ 2$ & $3.3 @ 10$ & $1.8 @ 1$ & $2.2 @ 1$ \\
\hline 5100018 & 2 & $1.0 @ 1$ & $3.6 @ 1$ & $1.0 @ 1$ & $1.6 @ 5$ & $1.4 @ 10$ & $1.4 @ 1$ & $3.6 @ 2$ \\
\hline 5175328 & 3 & $1.2 @ 1$ & $3.1 @ 1$ & $17.1 @ 2$ & $1.3 @ 1$ & $27.3 @ 1$ & $9.4 @ 1$ & $1.2 @ 1$ \\
\hline 5175323 & 5 & $4.2 @ 5$ & $13.0 @ 5$ & $11.4 @ 10$ & $1.5 @ 10$ & $9.7 @ 10$ & $5.5 @ 10$ & $2.2 @ 1$ \\
\hline 5145324 & 7 & $3.3 @ 1$ & $5.2 @ 1$ & $12.5 @ 2$ & $3.4 @ 10$ & $25.9 @ 2$ & $9.2 @ 2$ & $2.9 @ 1$ \\
\hline 5234881 & 3 & $1.2 @ 1$ & $1.5 @ 1$ & $1.8 @ 1$ & $1.4 @ 5$ & $2.7 @ 1$ & $4.5 @ 2$ & $2.2 @ 1$ \\
\hline 5238219 & 4 & $1.8 @ 5$ & $1.9 @ 2$ & $1.8 @ 2$ & $2.0 @ 10$ & $11.9 @ 10$ & $3.4 @ 5$ & $2.0 @ 2$ \\
\hline 5268029 & 0 & $1.4 @ 10$ & $1.7 @ 10$ & $1.3 @ 10$ & $1.3 @ 2$ & $1.3 @ 10$ & $1.3 @ 1$ & $1.7 @ 1$ \\
\hline Average SEM/me & ean & 0.036 & 0.064 & 0.027 & 0.016 & 0.039 & 0.047 & 0.041 \\
\hline \multicolumn{9}{|c|}{ 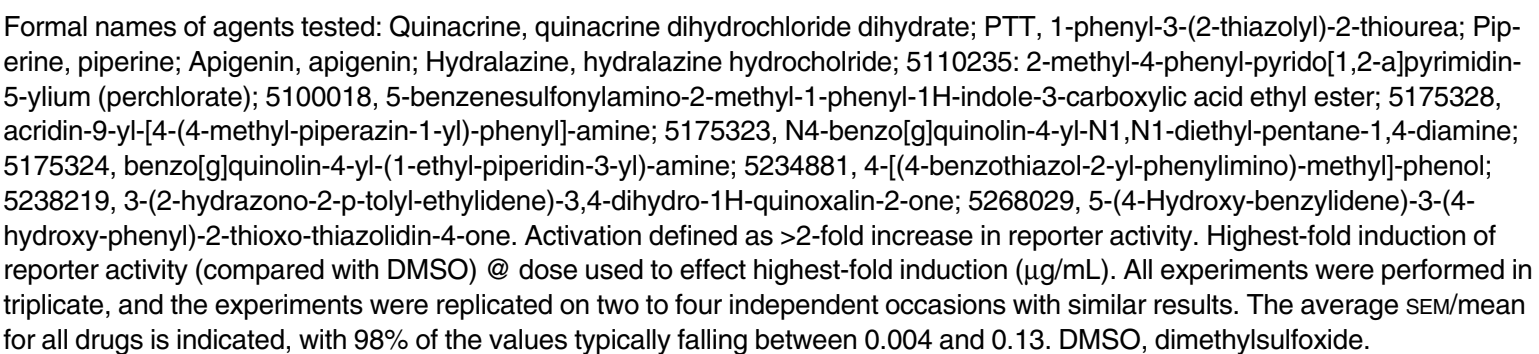 } \\
\hline
\end{tabular}

erence 6)]. Four of the 11 agents (36\%) were structurally similar (quinacrine, 5175323, 5175324, and 5175328 all share a similar heterotricyclic functional group that is known to intercalate into DNA), and these generally produced the largest induction of the reporter systems (Table 2). All assays were performed in triplicate, and all experiments were replicated independently on two to four separate study periods.

Unlike TSA and Scriptaid, this group of agents did not appear to have histone deacetylation as the mechanism of action, as evidenced by the relatively stable level of acetylated histones as assayed by immunoblot (data not shown). Some compounds produced minor changes in levels of methylhistone $\mathrm{H} 3$ and no apparent changes in phosphohistones (data not shown). Although a systematic evaluation of the mechanism of the agents' described effects was not undertaken, non-HDACI possibilities for nonspecifically facilitating expression include augmenting the activity of ubiquitous occult enhancer elements, DNA polymerases, RNA polymerase, mRNA-stabilizing proteins, and polysomes or by suppressing proteasomedependent proteolysis. We suspected that these agents would synergize with TSA due to a differing mechanism of action, and indeed several of them did.

To demonstrate this, we measured light emission from p53-R RKO cells after incubation with each test agent and escalating doses of TSA. The test agents were two compounds (5100018 or 5175323) or two drugs (quinacrine or apigenin) at a single dose. Varying doses of TSA with or without the single dose of compound were added to cells to construct a dose scale $(0,0.05,0.1$,
$0.2,0.4$, or $0.8 \mu \mathrm{g} / \mathrm{mL}$ TSA). A merely additive effect was observed when 5100018 was combined with TSA. For example, $2 \mu \mathrm{g} / \mathrm{mL} 5100018$ added to a given dose of TSA produced a similar increase as did an equipotent additional dose of TSA when tested at multiple points in the TSA dose scale. A robustly synergistic effect was seen however, when 5175323, quinacrine, and apigenin were added to TSA, again testing at multiple points along the scale. For example, a doubling of TSA alone from 0.05 to $0.1 \mu \mathrm{g} / \mathrm{mL}$ increased luciferase activity by approximately $100 \%$, but the addition of an equipotent dose of 5175323 to $0.05 \mu \mathrm{g} / \mathrm{mL}$ TSA resulted in an increase in luciferase activity by about $700 \%$, a 7 -fold increase that is evidence of a synergistic relationship. Similar synergistic effects were produced by quinacrine and apigenin. Syn- 


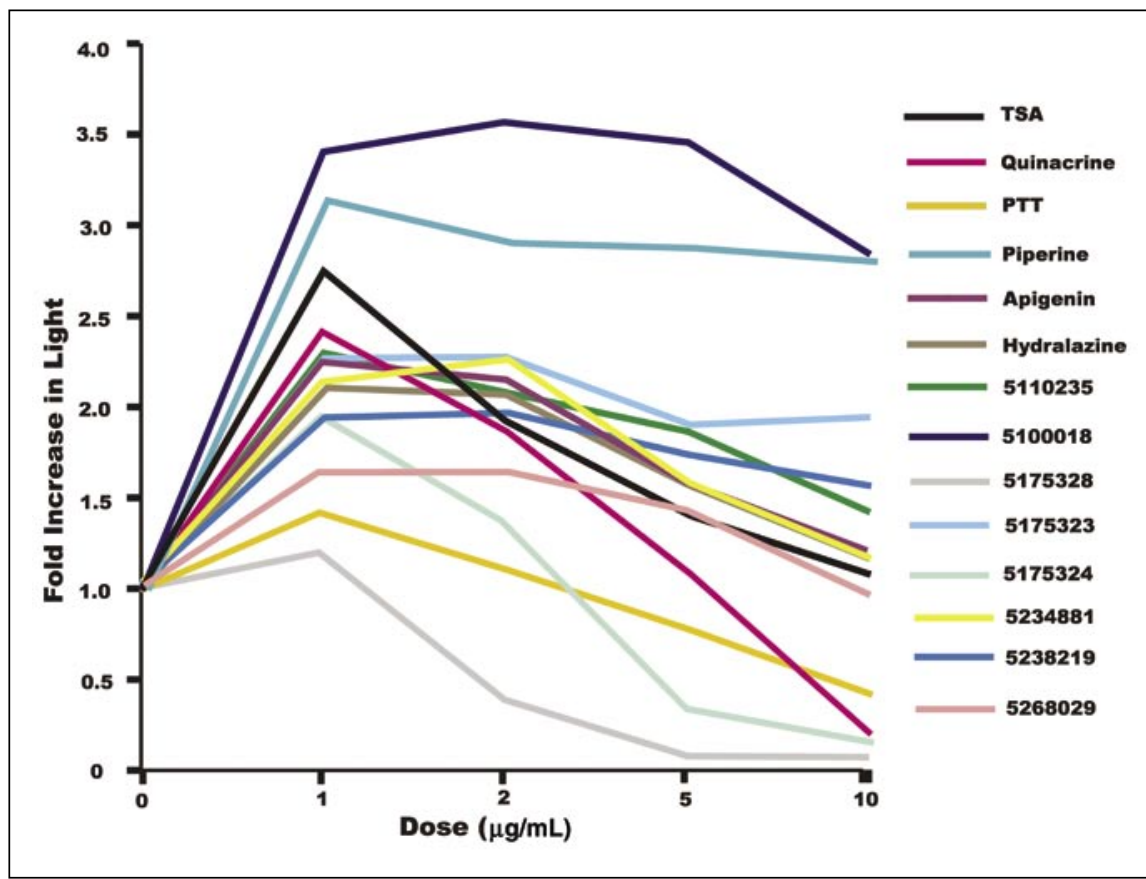

Figure 1. Response of the Renilla Shh-Light 2 reporter cells to a panel of expression facilitators. Data points represent the average of the readings of triplicate wells of a representative experiment. The average of the SEM/mean for all points was 0.041. TSA, trichostatin-A; PTT, 1-phenyl-3-(2-thiazolyl)2-thiourea.

ergy was maintained in the presence of $4 \mathrm{mM}$ caffeine for p53-R cells and replicated in the Smad4-R cells (data not shown), excluding $\mathrm{p} 53$ pathway activation (due to DNA damage) as an artifactual mimic of synergy. All synergy assays were performed in duplicate, and experiments were replicated on two or three separate study periods.

Some agents had optimal reporter effects at a concentration that was borderline toxic to cells, especially true of quinacrine and TSA (Figure 1) and of 5175323 and 5175324 in other reporter systems (data not shown). Although some transcriptional facilitators [e.g., the HDACI suberoylanilide hydroxamic acid (SAHA)] are already in clinical trials (10), the observation that expression facilitation is seen at near toxic levels generally reflects the established concern for the safety of the classes of compounds that would be expected nonspecifically to up-regulate transcription in patients. Other compounds (e.g., 5175328 in Figure 1) acted only to reduce reporter activity, and most of such compounds are presumed to be toxic.

Compound and drug libraries are commonly screened in search of novel therapeutics. Compound libraries can be expected to contain a group of agents nonspecifically modulating gene expression. This expectation will affect the planning and interpretation of such endeavors.

\section{ACKNOWLEDGMENTS}

We thank Dr. Bert Vogelstein for the DLDI/BFP cell line, Dr. Wafik El-Diery for the HCT116/p53-R cell line, and Dr. Philip Beachy for the Shh Light 2 cell line.

\section{REFERENCES}

1.Jensen, L.E. and A.S. Whitehead. 2003. ELAM-1/E-selectin promoter contains an inducible AP-1/CREB site and is not NF-kappa B-specific. BioTechniques 35:54-58.

2.Hong, S.J., H. Chae, and K.S. Kim. 2002. Promoterless luciferase reporter gene is transactivated by basic helix-loop-helix transcription factors. BioTechniques 33:1236-1240.

3.Osborne, S.A. and K.F. Tonissen. 2002. pRLTK induction can cause misinterpretation of gene promoter activity. BioTechniques 33:12401242.

4.Annicotte, J.S., K. Schoonjans, C. Haby, and J. Auwerx. 2001. An E-box in pGL3 reporter vectors precludes their use for the study of sterol regulatory element-binding proteins. BioTech- niques 31:993-996

5.McGovern, S.L., E. Caselli, N. Grigorieff, and B.K. Shoichet. 2002. A common mechanism underlying promiscuous inhibitors from virtual and high-throughput screening. J. Med. Chem. 45:1712-1722.

6.Su, G.H., T.A. Sohn, B. Ryu, and S.E. Kern. 2000. A novel histone deacetylase inhibitor identified by high-throughput transcriptional screening of a compound library. Cancer Res. 60:3137-3142.

7.Yoshida, M., M. Kijima, M. Akita, and T. Beppu. 1990. Potent and specific inhibition of mammalian histone deacetylase both in vivo and in vitro by trichostatin A. J. Biol. Chem. 265:17174-17179.

8.Sohn, T.A., R. Bansal, G.H. Su, K.M. Murphy, and S.E. Kern. 2002. High-throughput measurement of the Tp53 response to anticancer drugs and random compounds using a stably integrated Tp53-responsive luciferase reporter. Carcinogenesis 23:949-957.

9.Mulholland, N.M., E. Soeth, and C.L. Smith. 2003. Inhibition of MMTV transcription by HDAC inhibitors occurs independent of changes in chromatin remodeling and increased histone acetylation. Oncogene 22:4807-4818.

10.Kelly, W.K., V.M. Richon, O. O'Connor, T. Curley, B. MacGregor-Curtelli, W. Tong, M. Klang, L. Schwartz, et al. 2003. Phase I clinical trial of histone deacetylase inhibitor: suberoylanilide hydroxamic acid administered intravenously. Clin. Cancer Res. 9:3578-3588.

11.Wang, W. and W.S. El-Deiry. 2003. Bioluminescent molecular imaging of endogenous and exogenous p53-mediated transcription in vitro and in vivo using an HCT116 human colon carcinoma xenograft model. Cancer Biol. Ther. 2:196-202.

12.Torrance, C.J., V. Agrawal, B. Vogelstein, and K.W. Kinzler. 2001. Use of isogenic human cancer cells for high-throughput screening and drug discovery. Nat. Biotechnol. 19:940-945.

13.Gossen, M. and H. Bujard. 1992. Tight control of gene expression in mammalian cells by tetracycline-responsive promoters. Proc. Natl. Acad. Sci. USA 89:5547-5551.

14.Taipale, J., J.K. Chen, M.K. Cooper, B. Wang, R.K. Mann, L. Milenkovic, M.P. Scott, and P.A. Beachy. 2000. Effects of oncogenic mutations in Smoothened and Patched can be reversed by cyclopamine. Nature 406:1005-1009.

15.Wilson, V.S., K. Bobseine, C.R. Lambright, and L.E. Gray, Jr. 2002. A novel cell line, MDA-kb2, that stably expresses an androgenand glucocorticoid-responsive reporter for the detection of hormone receptor agonists and antagonists. Toxicol. Sci. 66:69-81.

Received 10 March 2004; accepted 25 March 2004.

Address correspondence to Scott E. Kern, Department of Oncology, Rm 461, Cancer Research Building, 1650 Orleans Street, Sidney Kimmel Comprehensive Cancer Center, The Johns Hopkins University School of Medicine, Baltimore, MD 21231, USA.e-mail: sk@jhmi.edu 